\title{
How Losing Sleep Following Vaccination May Weaken the Response to SARS-CoV Vaccines
}

\author{
Nasreen Akhtar $^{1}$ D $\cdot$ Rupesh K. Srivastava ${ }^{2} \cdot$ Deepak Shrivastava $^{3}$
}

Received: 4 January 2022 / Revised: 1 February 2022 / Accepted: 10 February 2022 / Published online: 17 February 2022

(c) The Author(s), under exclusive licence to Springer Nature Singapore Pte Ltd. 2022

\begin{abstract}
With the emergence of new SARS-CoV-2 variants, a close analysis of factors that affect the efficacy of the vaccine in different groups is a must. It is important to elucidate the role of clinical, behavioral and host factors on modulation of immunogenicity of the SARS-CoV-2 vaccine. Data from other vaccines have shown that duration and efficiency of sleep affect the immunogenicity of the vaccine. There is a need for identification of circadian influence and sleep on SARS-CoV-2 vaccine using validated immune correlates of protection. We propose that sleep acts as a natural adjuvant by promoting the immunological synapse formation between the antigen presenting cells and $\mathrm{CD} 4^{+} \mathrm{T}$ cells thereby leading to activation of cellular and humoral immunity in SARS-CoV-2 infection. Thus, apart from the titer of neutralizing antibodies, the cellular immunity including $\mathrm{CD} 4^{+} \mathrm{T}$ cells and memory $\mathrm{T}$ cells must be assessed to clearly demarcate the long-term effect of sleep duration and efficiency on vaccine immunogenicity.
\end{abstract}

SARS-CoV-2 vaccines do not elicit the same response in all individuals receiving them. Reports range between 90.3 and $97.6 \%$ for SARS-CoV-2 vaccine efficacy after the second dose [1]. However, vector produced by AstraZeneca has an efficacy between 41.0 and $75.2 \%$. The new variants of SARS-CoV-2 modify the vaccine efficacy of SARS-CoV-2 further. Mutations in the variants SARS-CoV-2 B.1.617.2 and B.1.1.529 may further alter the neutralization of SARS$\mathrm{CoV}-2$ by the vaccines as compared to the earlier variants [2]. Vaccine trials have established the baseline safety, efficacy, and immunogenicity of the vaccines, but the role of clinical, behavioral and host factors on modulation of the immunogenicity for the vaccines is now pertinent. Increasing age reduces immunogenicity [3, 4] but sex does not have a significant modifying role in immune response [1, 4], based on the emerging data from Phase III trials. On the other hand, the role of sleep in modification of the efficacy of the vaccines is likely significant.

Nasreen Akhtar

drnasreenakhtar@aiims.edu

1 Department of Physiology, All India Institute of Medical Sciences, Ansari Nagar, New Delhi 110049, India

2 Department of Biotechnology, All India Institute of Medical Sciences, New Delhi, India

3 Division of Pulmonary, Critical Care and Sleep, UC Davis School of Medicine, Sacramento, USA
Several studies have revealed that both duration and efficiency of sleep before and after vaccination against viral infections enhance the immune response. Lange et al. in 2011 , investigated the effect of sleep on immunological memory formation in 27 healthy men after vaccination against Hepatitis A. Only half the proportion of Hepatitis A-specific $\mathrm{CD}^{+} \mathrm{T}$ cells developed in participants who were awake in the night following Hepatitis A vaccination [5]. A reduction in immune response by more than $50 \%$ is reported in individuals who slept for less than four hours for four consecutive nights after vaccination against influenza [6]. Similarly, secondary antibodies were reduced in those with shorter sleep duration, as assessed by actigraphy, following hepatitis B vaccination [7]. There is some evidence for decreased adaptive cellular immunity against hepatitis A by poor-quality sleep in the nights following vaccination. Further, wakefulness in the night after vaccination against 2009 H1N1 influenza led to decreased specific antibodies in the early phase. The antibody titer after five days was reduced but it recovered at later time points.

Nocturnal sleep promotes an environment supportive to the activation of adaptive cellular immune response by modulation of IL-10 and IL-12 activity. [8] There is a bidirectional relationship between sleep and immune response (Fig. 1). Sleep acts as a natural adjuvant by promoting the immunological synapse formation between the antigen presenting cells and $\mathrm{CD} 4^{+} \mathrm{T}$ cells thereby leading 
Fig. 1 Mechanistic hypothesis: Sleep promotes secretion of $\mathrm{GH}$ and prolactin and suppresses cortisol, thus acting as an adjuvant for activation of naïve T cells. Release of IL-6, IL8, IFN $\gamma$, IL-1 $\beta$ and TNF $\alpha$ is enhanced during sleep, thus promoting the activation of $\mathrm{CD} 4+\mathrm{T}$ cells mediated activation of cellular and humoral immunity

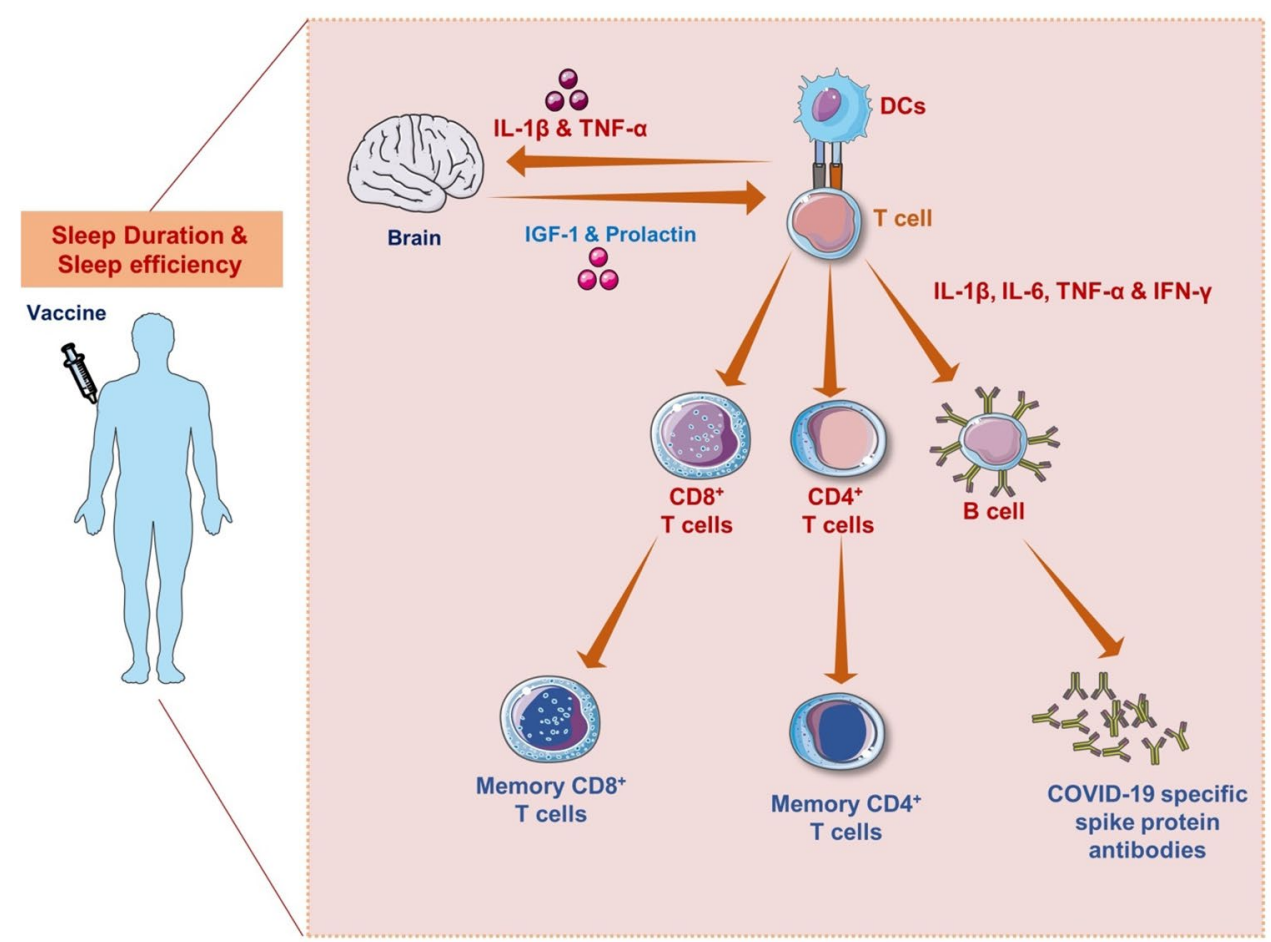

to activation of cellular and humoral immunity. [5] Upon activation of the immune system, inflammatory cytokines IL- $1 \beta$ and TNF- $\alpha$ cross the blood brain barrier and induce sleep. During sleep, growth hormone $(\mathrm{GH})$ viz. insulin growth factor-1 (IGF-1) and prolactin level is increased, and on the other hand cortisol secretion is suppressed. This creates a milieu conducive to activation of the helper cells, cytotoxic T cells, and B cells. SARS-CoV-2 specific spike protein antibodies will be produced by these activated $\mathrm{B}$ cells. $\mathrm{CD}^{+}$and $\mathrm{CD}^{+} \mathrm{T}$ cells will help in the formation of SARS-CoV-2 specific memory $\mathrm{T}$ cells to sustain long-term immunity against SARS-CoV-2. For most healthy individuals, variations in sleep around the vaccination days may not add up to affect the efficacy of the vaccine. In contrast, for immunocompromised individuals and for those with comorbidities, ensuring adequate sleep in the nights after vaccination may help generate an adequate protective response.

According to a recent commentary circadian influence including the time of the day the vaccine is injected may also modulate antibody production [9]. A study in 2008 suggested that in men, vaccination in the forenoon was associated with more antibody production [10]. More data is needed to understand the timing and immune response in subjects with phase-shift disorders.

There is a critical need for the identification of these factors using unbiased and objective assessments. The impact of the factors on host response must be assessed using validated immune correlates of protection. Most studies utilize the titer of neutralizing antibodies to characterize the vaccine induced protective immunity. Currently, non-human primate studies indicate that neutralizing antibodies targeting RBD of the virus is a potential immune correlate of protection, and cellular immune response is also vital for control of the virus. The cellular immunity must be assessed following vaccination, as reduced $\mathrm{CD}^{+} \mathrm{T}$ cells have been shown to partially abrogate the protective efficacy, especially when antibody titers are low. Durability of immune responses and true causation can only be established by longitudinal follow-up studies.

To maximize the effectiveness of SARS-CoV-2 vaccination across the world, it is important to delineate the individual factors which affect SARS-CoV-2 vaccination. Along with cross sectional data at particular time points and longitudinal analysis of all the immune correlates of protection is required. This data will eventually provide critical and actionable information for generating health policy, treating patients, and modulating vaccine efficacy.

\section{References}

1. Polack FP, Thomas SJ, Kitchin N, et al. Safety and efficacy of the BNT162b2 mRNA covid-19 vaccine. N Eng1 J Med. 2020;383:2603-15. https://doi.org/10.1056/NEJMoa2034577.

2. Planas D, Bruel T, Grzelak L, et al. Sensitivity of infectious SARS-CoV-2 B.1.1.7 and B.1.351 variants to neutralizing antibodies. Nat Med. 2021;27:917-24. https://doi.org/10.1038/ s41591-021-01318-5. 
3. Richards NE, Keshavarz B, Workman LJ, Nelson MR, Platts-Mills TAE, Wilson JM. Comparison of SARS-CoV-2 antibody response by age among recipients of the BNT162b2 vs the mRNA-1273 vaccine. JAMA Netw Open. 2021;4(9): e2124331. https://doi.org/ 10.1001/jamanetworkopen.2021.24331.

4. Abu Jabal K, Ben-Amram H, Beiruti K, et al. Impact of age, ethnicity, sex and prior infection status on immunogenicity following a single dose of the BNT162b2 mRNA COVID-19 vaccine: realworld evidence from healthcare workers, Israel, December 2020 to January 2021. Euro Surveill. 2021;26(6):2100096. https://doi. org/10.2807/1560-7917.ES.2021.26.6.2100096.

5. Benedict C, Cedernaes J. Could a good night's sleep improve COVID-19 vaccine efficacy? Lancet Respir Med. 2021;9:447-8. https://doi.org/10.1016/S2213-2600(21)00126-0.

6. Spiegel K, Tasali E, Penev P, et al. Brief communication: sleep curtailment in healthy young men is associated with decreased leptin levels, elevated ghrelin levels, and increased hunger and appetite. Ann Intern Med. 2004;141:846-50. https://doi.org/10. 7326/0003-4819-141-11-200412070-00008.

7. Prather AA, Hall M, Fury JM, et al. Sleep and antibody response to hepatitis B vaccination. Sleep. 2012;35:1063. https://doi.org/ 10.5665/sleep. 1990 .
8. Benedict C, Brytting M, Markström A, et al. Acute sleep deprivation has no lasting effects on the human antibody titer response following a novel influenza A H1N1 virus vaccination. BMC Immunol. 2012;13:1. https://doi.org/10.1186/1471-2172-13-1.

9. Druzd D, Matveeva O, Ince L, et al. Lymphocyte circadian clocks control lymph node trafficking and adaptive immune responses. Immunity. 2017;46:120-32. https://doi.org/10.1016/j.immuni. 2016.12.011.

10. Phillips AC, Gallagher S, Carroll D, et al. Preliminary evidence that morning vaccination is associated with an enhanced antibody response in men. Psychophysiology. 2008;45:663-6. https://doi. org/10.1111/j.1469-8986.2008.00662.x.

Publisher's Note Springer Nature remains neutral with regard to jurisdictional claims in published maps and institutional affiliations. 